\title{
Upgrade of the Magnetic Proton Recoil (MPRu) spectrometer for 1.5-18 MeV neutrons for JET and the next step
}

\author{
Göran Ericsson*, E.Andersson-Sundén, H.Sjöstrand, S.Conroy, M.Gatu-Johnson, \\ L.Giacomelli, C.Hellesen, A.Hjalmarsson, J.Källne, E.Ronchi, M.Weiszflog, \\ G.Wikström, \\ Dept. of Neutron Research, Uppsala university \\ Box 525, SE-751 20 UPPSALA, Sweden \\ E-mail: goran.ericsson@tsl.uu.se
}

\section{G.Gorini, M.Tardocchi,} INFM, Dept. of Physics, Univ. of Milano-Bicocca and Istituto di Fisica del Plasma (IFP-CNR) Milan, Italy

\section{R.C.Pereira, A.Combo, N.Cruz, J.Sousa, C.Correia,}

Centro de Fusão Nuclear, Instituto Superior Técnico

Av. Rovisco Pais 1, 1049-001 Lisboa, Portugal

\section{S.Popovichev and JET EFDA contributors ${ }^{\dagger}$}

JET, Culham Science Centre

Abingdon, UK

We describe the upgraded Magnetic Proton Recoil fusion neutron spectrometer (MPRu). The original MPR was installed at the JET fusion experiment in 1996, and was designed for diagnosis of the 14-MeV neutron emission in high-power deuterium-tritium (DT) fusion experiments. The goal of the MPR upgrade project is to widen the operational range of the instrument to the full range of fusion relevant neutron energies, $1.5<\mathrm{E}_{\mathrm{n}}<18 \mathrm{MeV}$, to increase its flexibility and to improve the background immunity of the spectrometer's focal plane detector. In this paper we describe how these goals have been achieved by the installation of a new detector hodoscope, based on phoswich scintillators, in combination with state-of-the-art digital data acquisition electronics and enhanced monitoring capabilities. Examples of test results and predicted performance are given.

International Workshop on Fast Neutron Detectors

University of Cape Town, South Africa

April 3-6, 2006

\footnotetext{
${ }^{*}$ Speaker

$\dagger$ See annex of J. Pamela et al, "Overview of JET Results “, Proc. $20^{\text {th }}$ IAEA Fusion Energy Conference, Vilamoura, Portugal (2004)
} 


\section{Introduction}

Present high-performance fusion energy experiments, such as JET, operate with fuels of pure deuterium (D) and, on special occasions, mixtures of deuterium and tritium (DT). In such experiments, both of the neutron-producing fusion reactions $d+d \rightarrow{ }^{3} \mathrm{He}+n\left(E_{n}(0)=2449 \mathrm{keV}\right)$ and $\mathrm{d}+\mathrm{t} \rightarrow{ }^{4} \mathrm{He}+\mathrm{n}\left(\mathrm{E}_{\mathrm{n}}(0)=14028 \mathrm{keV}\right)$ will occur. In the case of "pure" D plasmas a component of $14-\mathrm{MeV} \mathrm{dt}$ neutrons is present due to the tritium produced in the breeding reaction $\mathrm{d}+\mathrm{d} \rightarrow$ $\mathrm{t}+\mathrm{p}$. In mixed DT operations, $\mathrm{d}+\mathrm{d} \rightarrow{ }^{3} \mathrm{He}+\mathrm{n}$ reactions will also take place, albeit at a muchreduced rate compared to the dominant $\mathrm{dt}$ fusion. It is the role of fusion Neutron Emission Spectroscopy (NES) to exploit the information carried by the emission of these 2.5 and $14-\mathrm{MeV}$ neutrons. A wealth of information about the fusion process can be extracted from the neutron emission, e.g., the fusion power, the fuel ion thermal temperature, the presence and intensity of various supra-thermal ion populations, the collective motion of the fusion plasma etc.[1]; NES is in this respect a truly multi-parameter diagnostic. The design of a high-performance NES system must be governed by the particular requirements of this measurement situation.

In this paper we describe a spectrometer for diagnosis of the neutron emission in fusion experiments based on the Magnetic Proton Recoil (MPR) technique. The design of the instrument follows that of similar instruments built for high-performance spectroscopy in nuclear physics. However, the system presented here has been specifically designed to meet the requirements of fusion neutron measurements in terms of resolution, efficiency, calibration, sensitivity, reliability and robustness. The original MPR was installed at JET in 1996 and was intended for 14-MeV measurements in high-power DT operations [2]. It was successfully operated during the major DT experimental campaign DTE1 in 1997 and in the Trace Tritium campaign of 2003. However, tritium operations are rare and to widen the operational range of the instrument an upgrade project, dubbed MPRu, was initiated with the goal to allow measurements also of the $2.5-\mathrm{MeV}$ neutron emission from $\mathrm{D}$ plasmas. The upgrade project was undertaken as part of a programme for Enhanced Performance at JET, JET-EP1. The installation of the MPRu was completed in the end of 2005. This paper describes the general MPRu principle and its components and then discusses in more detail some of the upgraded systems, such as the new focal plane detector, the data acquisition system and the control and monitoring system. Some examples of test results and simulations of the expected spectrometer performance are also given.

\section{The MPR principle}

The principle and components of the Magnetic Proton Recoil technique are illustrated in Fig. 1. Neutrons from the reactor are formed into a "beam" by a neutron collimator. Some of these neutrons undergo elastic nuclear collisions with the hydrogen nuclei (protons) of a thin plastic foil ("target") placed in the neutron beam, resulting in the emission of recoil protons. Recoil protons emitted in the forward direction (and thus of nearly the same energy as the original neutrons) are allowed to enter the magnetic part of the spectrometer through a circular proton collimator. The selected protons are momentum analyzed in the magnetic field and focused 
onto the focal plane, well removed from the neutron beam. A multi-element focal plane detector counts the spatially dispersed protons, providing the spectrometer's basic data as time-resolved proton position histograms. These histograms can be related back to the original energy distribution and rate of neutrons on the target foil by the well-known spectrometer response function.

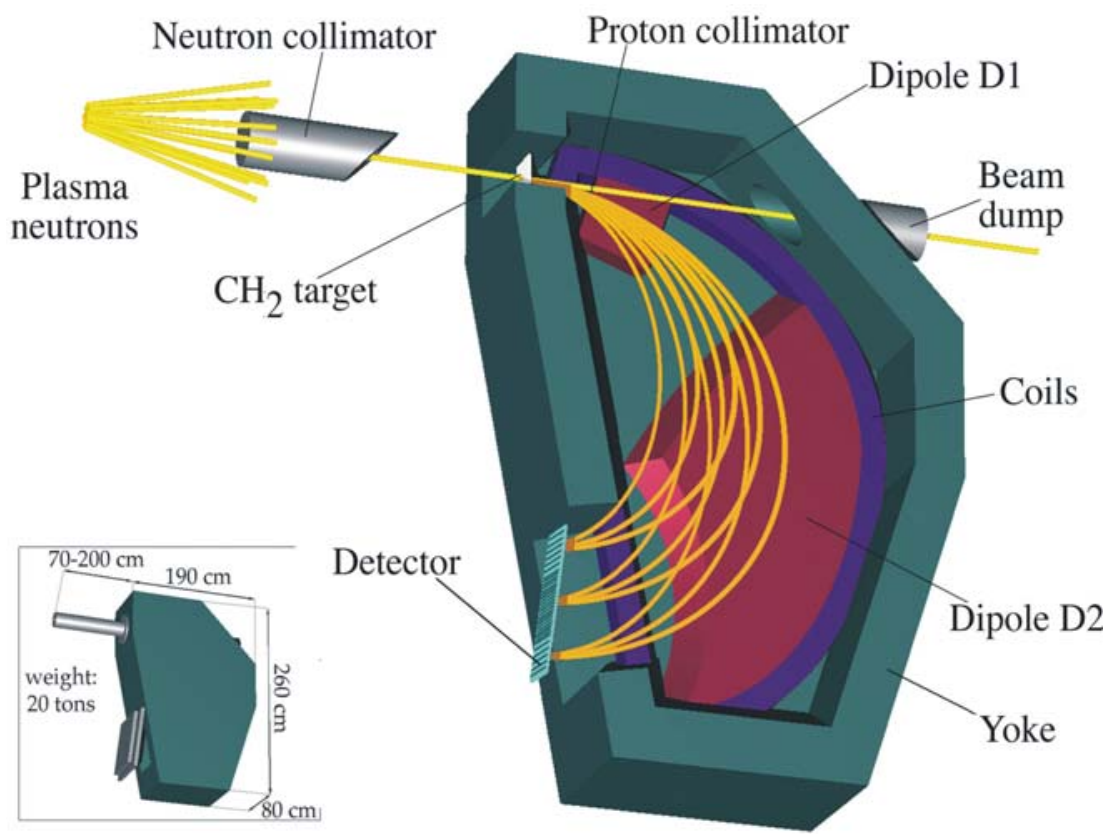

Fig. 1. Schematic figure of the MPRu spectrometer, without its radiation shield.

Both neutron-to-proton "conversion" in the target and proton energy (momentum) determination are done passively, only the counting of protons at the focal plane is an active measurement. The selection of protons in the forward direction has several advantages: first, it maximizes the recoil proton energy, which improves the signal-to-background (S:B) ratio in the detector; second, the $\mathrm{H}(\mathrm{n}, \mathrm{p})$ cross section is maximized; and third, it minimizes the contribution of the kinematical shift to the energy resolution. The count rate limitation of the system now rests only with the proton counter at the focal plane; counting protons with scintillators can be done at great rates and efficiencies so that the maximum rate capacity of the full system is high, $\mathrm{C}_{\text {cap }}>>\mathrm{MHz}$. Furthermore, the calibration of such a system depends only on well-known physical constants (e.g., stopping powers and cross sections), well-understood physics (of electromagnetism and charged-particle motion in magnetic fields etc.), the magnetic field topology and level and, finally, the geometry and alignment of the instrument. Magnetic (B) fields and geometry can be measured to great accuracy and hence an $a b$ intio absolute calibration of the spectrometer can be established both in terms of energy and efficiency.

The implementation of this technique in the MPRu has focused on high immunity to background (Sect. 3), calibration accuracy (Sect. 5) and flexibility. Thus, the neutron collimator, a steel cylinder of $10 \mathrm{~cm}^{2}$ bore, is adjustable from a minimum $70 \mathrm{~cm}$ up to $200 \mathrm{~cm}$. Six different target foils (all $10 \mathrm{~cm}^{2}$ of chemically pure $\mathrm{CH}_{2}$ ) are available by remote control, spanning a thickness range of $1.5-23 \mathrm{mg} / \mathrm{cm}^{2}$. Three different proton apertures are installed, also remotely 
controlled, providing solid angles of 10, 40 and $52.5 \mathrm{msr}$ into the spectrometer. Finally, the electro-magnet can be tuned to any neutron (recoil proton) energy between 1.5 and $18 \mathrm{MeV}$. The flexibility offered by these variable settings allows the measurement emphasis to be put on either resolution or count rate, as dictated by the general experimental conditions. The optimized reference setting for $14-\mathrm{MeV}$ measurements, using a $8-\mathrm{mg} / \mathrm{cm}^{2}$ foil and the $40 \mathrm{msr}$ collimator, gives a flux efficiency of $0.5 \cdot 10^{-4} \mathrm{~cm}^{2}$ with a near-Gaussian instrumental line shape of $2.5 \%$ resolution (FWHM); this corresponds to the width of the neutron emission of a $4 \mathrm{keV}$ thermal DT plasma. Corresponding conditions in $2.5-\mathrm{MeV}$ measurements give a somewhat relaxed resolution requirement (6.7\%). Background suppression is done in several steps: first, the spectrometer is completely enclosed in a 65-ton concrete radiation shield, with an extra 2ton lead shield around the detector box for enhanced $\gamma$ protection; second, the detector is removed from the neutron beam by about $1.5 \mathrm{~m}$; third, the detector scintillators are quite insensitive to neutral background like neutrons and gammas; and fourth, the use of the phoswich technique for the hodoscope elements further separates signal (protons) from background. Finally, background corrections based on post-processing can also be applied.

\section{The MPRu focal plane detector}

The focal plane detector of the MPRu is a straight-plane 32-channel scintillator (phoswich) hodoscope of about $52 \mathrm{~cm}$ length in the magnet's dispersive plane, as depicted in Fig. 2. This corresponds to an energy bite of about $\pm 20 \%$ around the central energy (chosen by the setting of the B-field). There are three hodoscope sections with different dimensions of the scintillators in each section. At the low-energy side is a 9-element section of $20 \mathrm{~mm}$ wide scintillators, in the centre a 13-element section of $10 \mathrm{~mm}$ wide detectors, and finally a 10-element high-energy section of $20 \mathrm{~mm}$ wide detectors; all elements are separated by a nominal $0.5-\mathrm{mm}$ gap and all are $10 \mathrm{~cm}$ long. The detector elements are wrapped in thin $(6 \mu \mathrm{m}) \mathrm{Al}$ foil to prevent cross talk and improve light collection, while minimizing the energy loss of the incoming proton recoils.
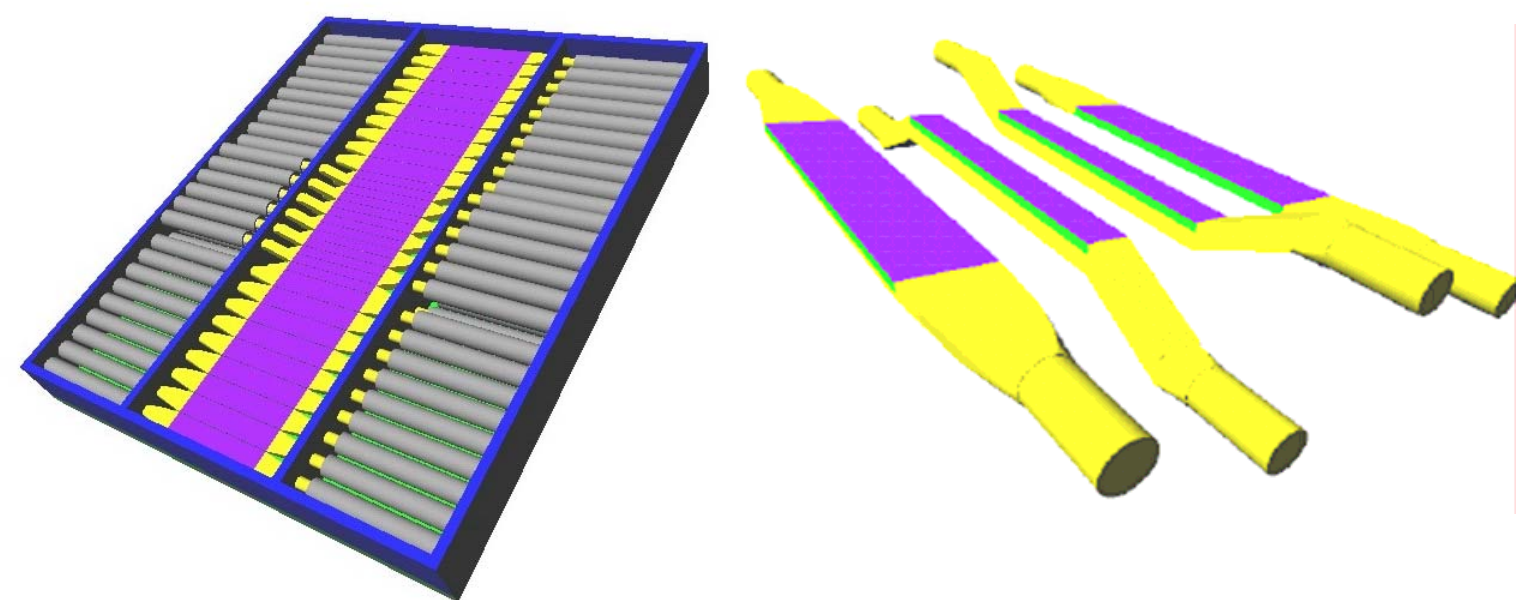

Fig. 2. Left: schematic view of the fully assembled MPRu hodoscope. Right: picture of the different scintillator - light guide combinations used: two 20-mm wide detectors of different thickness to the sides, and two 10-mm wide detectors of same thickness but with different light guide arrangements in the middle. Fast scintillator (purple), slow scintillator (green), light guide (yellow), PM tube (grey) and mechanical support (blue). 
The relative positions of the 32 phoswich elements in the hodoscope were determined to $50 \mu \mathrm{m}$ precision using a dedicated scanning system based on illumination with UV light through a narrow optical fibre. The position of the hodoscope with respect to the rest of the spectrometer was determined using the JET digital photographic surveying system, as described below.

To allow measurements of both the 2.5 and $14-\mathrm{MeV}$ neutron emission (separately), each of the 32 elements of the hodoscope consists of a two-layered phoswich detector. The two layers are in optical contact, but have distinctly different timing properties, making it possible to distinguish particles of different energies and types by the characteristic pulse shapes they generate. In the MPRu application, facing the incoming signal protons is a thin layer of fast BC404 (1.8 ns decay time), to which is attached a thick layer of slow BC444 (180 ns decay time). The thickness of the thin layer is $0.3 \mathrm{~mm}$ for all elements. Recoil protons with energies up to 4.7 $\mathrm{MeV}$ are stopped in this layer, including those of $2.5 \mathrm{MeV}$ from measurements of dd fusion reactions. This also gives a comfortably large " $\Delta \mathrm{E}$ " signal for penetrating protons when the spectrometer is set for measurements at higher neutron energies. The total thickness of the phoswich elements differs between the three detector sections; the high-energy section stops protons up to $18 \mathrm{MeV}$. A schematic picture of the different phoswich elements is shown in Fig. 2 , where is also shown the two different light guide arrangements for the $10-\mathrm{mm}$ wide detectors.

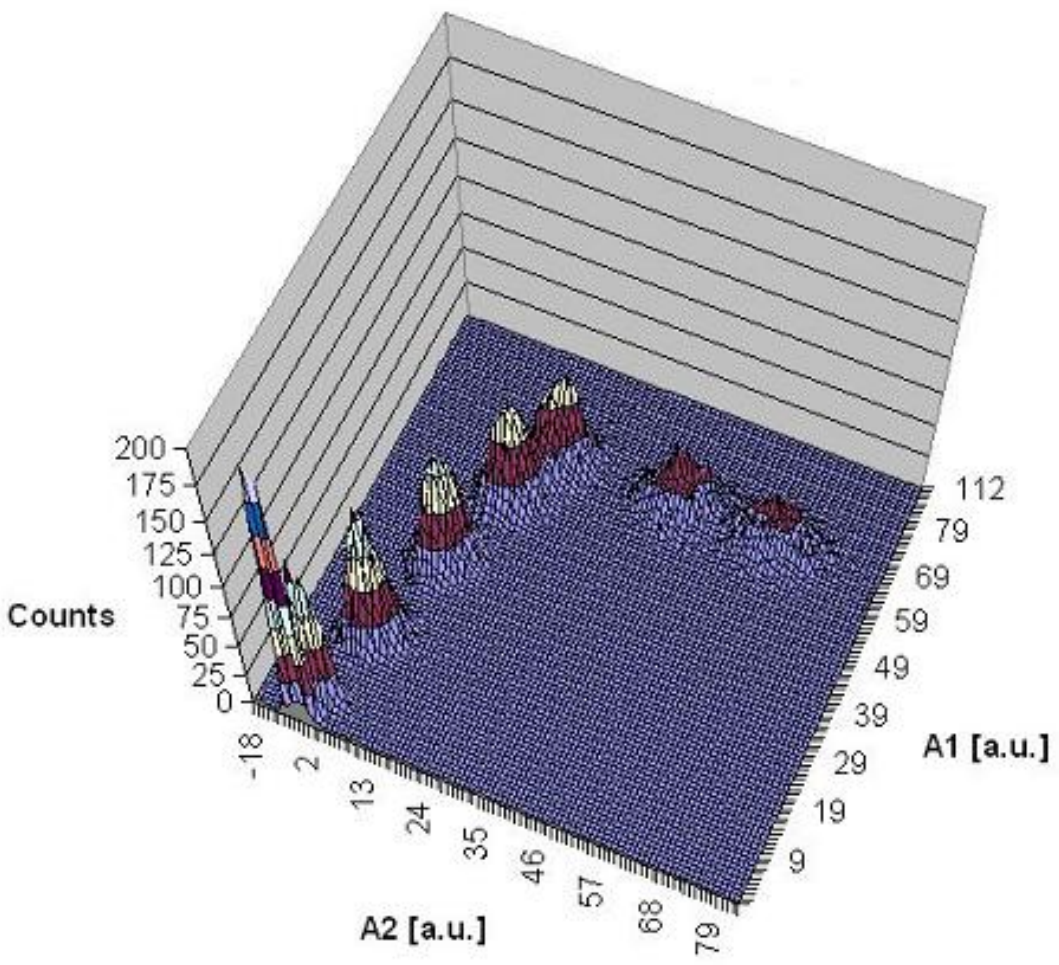

Fig. 3. Plot representing data for protons impinging on a test phoswich detector, acquired at the Uppsala Tandem Accelerator facility. A standard pulse shape discrimination technique has been used, integrating wave forms over an early, short gate (A1) and a late, long gate (A2). The peak at the lower left corner correspond to data for pick-up noise, while the other peaks, from left to right, correspond to data for protons of energies 2, 3, 4, 4.7, 6, and $7 \mathrm{MeV}$, respectively. 
Each phoswich element has two photomultiplier (PM) tubes attached via light guides (Fig. 2). These collect the emitted scintillation light and transform it to electrical signals, which are transmitted to the data acquisition system for processing and storage. The use of two PM tubes per phoswich element increases the total light collection and gives quite uniform longitudinal light collection efficiency.

Extensive tests have been performed on the phoswich detectors to study their performance in terms of resolution, longitudinal light collection efficiency, pulse shape characteristics etc. In these tests were used radioactive sources emitting $\alpha$ and $\beta$ particles, proton beams from the Uppsala Tandem accelerator, as well as artificial light sources such as light emitting diodes (LED) and a laser. The tests were performed to check the performance and quality of individual detector elements, to give input to the analysis and simulation programs and in general to study the suitability of the phoswich technique for this application. As an example of such test, the separation of protons of energies between 2 and $7 \mathrm{MeV}$ is shown in Fig. 3. For this plot, the standard pulse shape discrimination technique of integrating the PM tube signal in a short, early and a long, late time gate has been applied. Protons up to $4.7 \mathrm{MeV}$ give most of their signal in the early gate, while higher-energy protons penetrate into the thick, slow scintillator layer and give increasingly more of their signal in the late gate.

The hodoscope is equipped with three different light sources to monitor the gain of the PM tubes (see next section for details). Light from an external LED or laser is transmitted to each scintillator via optical fibre. A fibre is attached to the back side of each detector; a small $(1 \mathrm{~mm}$ diameter) spot of diffuse, white paint is applied to the opposite side of the scintillator to improve the dispersion of light from the fibre to the PM tubes.

\section{Data acquisition and Control \& Monitoring system}

The electrical pulses from the two PM tubes attached to a phoswich element are summed and amplified in a set of custom-built Pulse Summing Amplifiers, placed on the platform behind the radiation shield. Summed signals for each detector element are transmitted via $120 \mathrm{~m}$ long cables to JET's Diagnostics Hall where the rest of the data acquisition electronics is situated.

Eight custom-built PCI Transient Recorder (TR) cards build up the core of the MPRu data acquisition system [3]. Each card has 4 input channels that sample the summed PM tube pulses at a resolution of 8 bits per sample and a rate of 200 mega-samples per second. The input range is 0 to $-1 \mathrm{~V}$, and both the trigger level (in steps of $4 \mathrm{mV}$ ) and an optional voltage offset can be set individually. Other flexible settings include the number of samples per waveform (up to $1024)$ and the number of pre-trigger samples. Waveforms and trigger times are stored in the 512-MB on-board memory during a plasma discharge and transferred to computer mass storage only after the active measurement phase. The fast on-board processing capacity of the cards makes real-time data processing possible. The use of TR boards to store the full information for each event gives a large degree of flexibility in the analysis. Baseline restoration, pile-up rejection or resolution and efficient pulse shape discrimination techniques can be applied.

The purpose of the MPRu control and monitoring system is to keep track of short- and long-term changes in the settings and environmental parameters of the system. A primary con- 
cern in this context is the gain of the PM tubes, since these are known to exhibit both loaddependent transients as well as more gradual effects of use and ageing. Therefore, a system with three different light sources is used to monitor time-dependent variations in the PM tube gain. An LED-based system is used for transient (level of 0.1s) and medium-term changes. A custombuilt LED driver gives a large degree of stability of the signal and offers flexibility in setting the rate, shape and intensity of the output light pulse; a $100 \mathrm{~ns}$ (FWHM), quasi-square pulse at a rate of about $100 \mathrm{~Hz}$ is used for the MPRu. The particular shape and fixed rate of this pulse makes it possible to feed it into the normal data stream of scintillator pulses. Due to their great short-term stability LED pulses offer a useful tool for monitoring transient changes such as those associated with variable and high count rates. An example of a LED pulse is shown in Fig. 4, which also illustrates the advantage in storing the full pulse shape for post-processing; here the improvement after baseline restoration is shown. The LED and its driver are situated on the rear platform, and the light is transmitted to the scintillators by optical fibre. To monitor the stability of the LED, an absolute reference is used in the form of a YAP:Ce scintillator with an embedded ${ }^{241} \mathrm{Am} \alpha$ source; two such sources are available, each mounted directly on the PM tube photocathode of detector elements 0 "left" and 0 "right" respectively. In addition, a high repetition rate laser is also available.

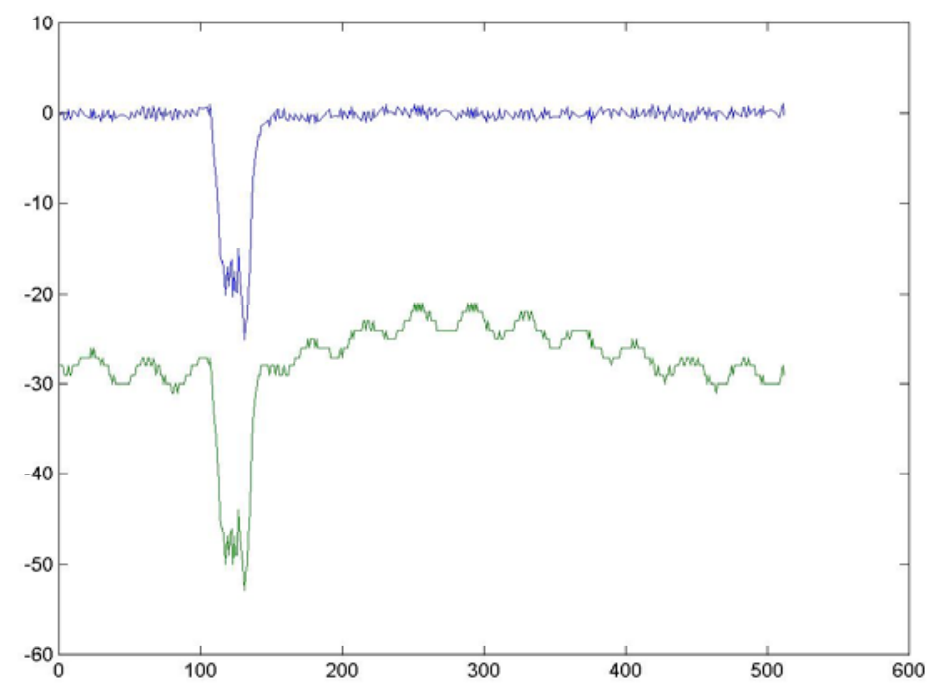

Fig. 4. Pulse form of a LED event registered by scintillator channel 0 , left side PM tube. The lower trace shows the unprocessed TR data as stored by the acquisition system, the upper trace shows the situation after baseline restoration. Vertical scale is voltage in units of $4 \mathrm{mV}$, horizontal scale is time in units of $5 \mathrm{~ns}$.

A number of probes are installed to measure the environmental parameters of the system. There are three Hall probes for monitoring B-fields and temperatures both in the electro-magnet and the detector. The vacuum level, various other temperatures, the PM tubes high-voltage settings, the settings and status of the electro-magnet power supply, the selected target foil and proton collimator are all continuously monitored. These data, including those from the TR cards, are finally stored with the JET central data acquisition system, CODAS. 


\section{Calibration and performance}

Calibration of magnetic spectrometers depends on two factors, beside the fundamental physics of electro-magnetism: first, accurate determination of the magnetic field topology at the excitations used experimentally, and second, precise alignment and surveying of the instrument. Mappings of the MPRu magnetic field were performed as part of the original installation at JET in 1996 [2]. Since the magnetic configuration is still the same, this original work is still valid, as are also the validation measurements performed with $\alpha$ particles and fusion neutrons.

The goal of the MPRu energy calibration effort is $\Delta \mathrm{E} / \mathrm{E}<10^{-4}$, which translates to a surveying accuracy of $0.1 \mathrm{~mm}$. Such accuracy was not achieved for the original MPR, and therefore a new detailed survey was conducted as part of the MPRu assembly. The survey was taken with JET's digital photography system, which could give the complete geometry of the MPRu installation in one common frame of reference. Based on the surveying results for a set of precisely known reference targets, it was concluded that this method is accurate to within about $50 \mu \mathrm{m}$ (over $0.5 \mathrm{~m}$ distances). Thus, in combination with the precise determination of the relative positions of the detector elements of the hodoscope, absolute positions of the detector and the spectrometer's line-of-sight (LOS) are determined to an accuracy well below the targeted 0.1 $\mathrm{mm}$. This corresponds to an absolute energy calibration of $\Delta \mathrm{E} / \mathrm{E}<1 \mathrm{keV} / 14 \mathrm{MeV}$.

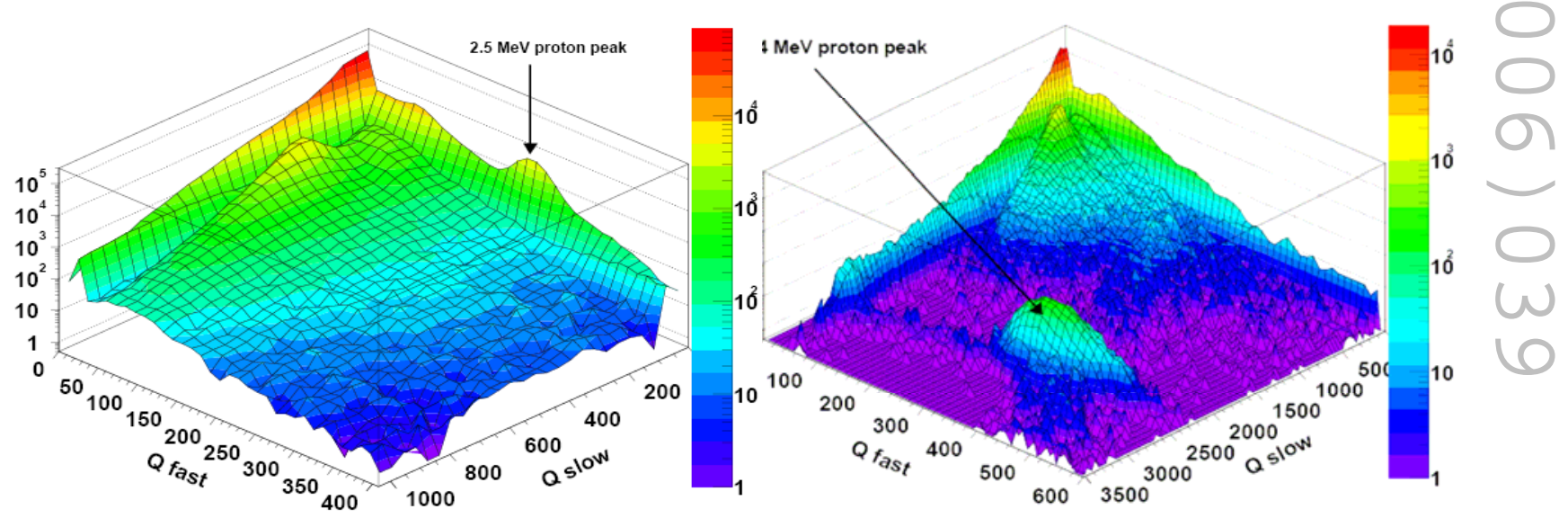

Fig. 5. Simulated signal (protons) and background for measurements of 2.5-MeV neutrons in a $\mathrm{D}$ plasma (left) and 14-MeV neutrons in a DT plasma (right). The $Q_{\text {fast }}$ and $Q_{\text {slow }}$ correspond to the early and late time gates of Fig. 3. Data are from simulations giving $10^{19} \mathrm{n}$ from thermal $\mathrm{D}$ and $\mathrm{DT}$ plasmas of $10 \mathrm{keV}$, respectively.

The absolute calibration in flux efficiency, important when using the spectrometer in measurements of the fusion power, depends on factors that in all relevant details are the same as for the original MPR system. Therefore, the earlier estimate of a $2.5 \%$ absolute systematic uncertainty in the spectrometer flux efficiency is still valid [4].

A Monte Carlo simulation model has been set up for the MPRu hodoscope, based on the GEANT4 package. The model includes the materials and geometry of the final installation, and also includes relevant results from the phoswich characterization measurements. This code can be used to predict the spectrometer's performance, optimize the analysis algorithms, explain 
observed phenomena and assist in possible future modifications of the hodoscope. Based on the types and intensities of background events observed with the original MPR system, the simulation model was used predict the performance of the new phoswich-based hodoscope in 2.5 and 14-MeV neutron measurements at JET. Results from such calculations are shown in Fig. 5. These results indicate that the signal-to-background situation will improve by at least a factor 100 in measurements of the $2.5-\mathrm{MeV}$ neutrons in D plasmas (to a level 10:1), and by at least a factor 20 in measurements of the 14-MeV emission from DT plasmas (to 20000:1).

\section{Discussion and conclusions}

The MPRu system has been specifically designed for high-quality NES measurements of both the $2.5-\mathrm{MeV}$ neutron emission from $\mathrm{D}$ plasmas and the $14-\mathrm{MeV}$ emission from DT plasmas. Spectroscopic measurements of the neutron emission from fusion plasmas require a high degree of flexibility from the diagnostic instrument. Therefore, the MPRu design allows for an experimental emphasis on either resolution or count-rate, permitting measurements for a wide range of plasma conditions, from relatively low-power $\mathrm{D}$ plasmas (giving count rates of $\mathrm{kHz}$ for $\left.10^{16} 2.5-\mathrm{MeV} \mathrm{n} / \mathrm{s}\right)$ to DT plasmas of the highest power (10 MHz for $\left.10^{19} 14-\mathrm{MeV} \mathrm{n} / \mathrm{s}\right)$. The design also permits an accurate absolute calibration in both energy and flux efficiency, making the instrument an independent monitor of important plasma parameters, such as the collective plasma rotation and the output fusion power, besides the information provided on the state of the fuel ions. The emphasis of the upgrade work has been to achieve a high level of background immunity in all measurement scenarios, where new possibilities have been added by the combined use of phoswich detectors and state-of-the-art digital data acquisition electronics (Transient Recorders). Simulations predict that measurements of $14-\mathrm{MeV}$ neutrons from DT plasmas will be virtually background-free and only the counting statistics will set the limit for the instrumental sensitivity. In measurements of the $2.5-\mathrm{MeV}$ neutron emission, $\mathrm{S}: \mathrm{B}$ is lower but the absolute character of the MPRu data will make an important contribution, due to its unique LOS and, in particular, as reference for calibration of other neutron instruments. In addition, with its narrow line width and high immunity to background, the MPRu can efficiently separate the scattered and direct neutron flux, thereby providing important data in the "intermediate" energy region $4-11 \mathrm{MeV}$, useful for benchmarking of neutron transport codes; this is a type of measurement that few other instruments can provide. These properties, and the general multiparameter nature of NES measurements, also make the MPRu technique an interesting option for the next step in fusion energy research, such as ITER.

\section{References}

[1] L.Giacomelli et al., Nuclear Fusion 45 (2005) 1191

[2] G.Ericsson et al., Rev. Sci. Instr 72 (2001) 759.

[3] A.Combo et al., Proc. $4^{\text {th }}$ IAEA TM on data acquisition, control etc. in fusion experiments, San Diego, USA, 2003.

[4] H.Sjöstrand et al., Proc. 31 EPS Conf. on Contr. Fusion and Plasma Physics, London, UK, 2004 